\title{
LEVANTAMENTO DA ARBORIZAÇÃO URBANA PÚBLICA DE MATA/RS
}

Cristiane Richter ${ }^{1}$, Marcia Xavier Peiter ${ }^{2}$, Adroaldo Dias Robaina², Angélica Rossana Castro de Souza ${ }^{3}$, Rafael Camargo Ferraz ${ }^{4}$, Alessandro Figliero de David ${ }^{5}$

\section{RESUMO}

Arborização urbana diz respeito aos elementos vegetais de porte arbóreo, dentro da cidade. Nesse enfoque, as árvores plantadas em calçadas, fazem parte da arborização urbana assim como parques e praças. As árvores possuem uma importância na qualidade de vida das pessoas e também uma função na conservação da biodiversidade no meio urbano. Assim, esse trabalho teve como objetivo realizar um levantamento florístico, incluindo árvores, arvoretas e palmeiras presentes nas ruas e praças da cidade de Mata/RS, a fim de fornecer informações essenciais para ajudar os gestores em processos de decisão. Para isso, foi realizado um levantamento de dados através de visitas in loco. A identificação das árvores foi realizada de maneira direta e imediata sempre que possível, utilizando para isso bibliografias específicas de apoio. Para quantificar a diversidade de espécies optou-se por analisar dois componentes, densidade de espécies e uniformidade, respectivamente, através do índice de Margalef e índice de Pielou. Observou-se a predominância de Patagonula americana L. e Lagerstroemia indica Lam., ambas ultrapassaram o limite sugerido. Notou-se a necessidade de um melhor planejamento da arborização e um remodelamento do aspecto paisagístico da cidade.

Palavras-chave: árvores, ruas, praças, plantas ornamentais

\section{SURVEY ABOUT THE URBAN FORESTRY IN MATA CITY/RS}

\begin{abstract}
Urban forestry concerns to species with arboreal features that grow within a city. From this point of view, trees planted on sidewalks are part of urban forests, as well as parks and public squares. Trees are important for people's life and also to maintain the biodiversity amid the urban environment. So this study aimed at realizing a floristic survey, including trees, small trees and palm trees planted on streets and parks of Mata city / RS, in order to provide the public managers essential information to help them in decision making processes. In order to accomplish this, a data survey was realized through visits in loco. The trees identification was made visually and immediately whenever it was possible, using specific bibliographies as a basis to do so. In order to quantify the diversity of species, it was taken into consideration two elements: the density of species and their uniformity, which were respectively analyzed through the Margalef's diversity index and Pielou's evenness index. There was found the predominance of Patagonula americana L. and Lagerstroemia indica Lam.; both species exceeded the suggested limit. It was also noticed the need of a better urban forestry planning as well as improving the landscape aspects of the city.
\end{abstract}

Key-words: trees, streets; parks; ornamental plants.

\footnotetext{
${ }^{1}$ Lic. Ciências Biológicas

2 Engenheiro Agrônomo, Dr.,Prof.Depto Engenharia Rural - UFSM, Avenida Roraima n 100, 97105-900. Santa Maria/RS - diasrobaina@gmail.com

${ }^{3}$ Lic. Ciências Biológicas, Mestranda em Geomática - UFSM, Avenida Roraima n 100, 97105-900. Santa Maria/RS - angelsoubio@gmail.com

${ }^{4}$ Lic. Matemática, Aluno de Doutorado em Engenharia Agrícola - UFSM, Avenida Roraima n 100, 97105-900. Santa Maria/RS - rafacferraz@gmail.com

${ }^{5}$ Lic. Ciências Biológicas, Mestranda em Biodiversidade Animal - UFSM, Avenida Roraima n 100, 97105-900. Santa Maria/RS - afd_david@hotmail.com
} 


\section{INTRODUÇÃO}

As árvores nos centros urbanos exercem funções importantes, sendo responsáveis por uma série de benefícios ambientais e sociais que melhoram a qualidade de vida nas cidades e a saúde física e mental da população. Arborizar uma cidade não significa apenas plantar árvores em ruas, jardins e praças, criar áreas de recreação pública e proteger áreas verdes particulares (RIBEIRO, 2009).

De acordo com Volpe-Filik et al. (2007), as árvores possuem uma capacidade única de controlar muitos dos efeitos adversos do meio urbano, contribuindo significativamente na melhoria da qualidade de vida.

Conscientes da importância da arborização urbana como elemento fundamental para a qualidade de vida da população, muitas prefeituras municipais têm procurado compatibilizar o desenvolvimento e a expansão urbana com a preservação de seu patrimônio histórico, paisagístico e ambiental, aí incluindo parques, praças, jardins e outras áreas verdes (GONÇALVEZ e ROCHA, 2003).

Porém para Silva et al. (2008), a maioria das prefeituras dos municípios brasileiros não se preocupa com o planejamento da arborização, o que leva os próprios moradores, muitas vezes, a realizarem o plantio nas áreas públicas. Desse modo, o padrão observado em muitas cidades brasileiras é de uma arborização irregular, inadequada e descontínua (SILVA et al., 2008).

Para Silva et al. (2002), essa situação é traduzida em futuros transtornos à população local, causando prejuízos como rompimento de fiação de energia elétrica e telecomunicação, entupimento de calhas, danos às redes subterrâneas de água e de esgoto, obstáculos para circulação e acidentes envolvendo pedestres, veículos ou edificações. Tais problemas começam ainda na falta de tratos culturais para a produção de mudas destinadas à arborização (GONÇALVES et al., 2004).

De acordo com Moura e Santos (2009) o sucesso do planejamento da arborização está na escolha da espécie adequada a cada lugar. Uma árvore mal escolhida pode significar gastos por um longo tempo com manutenção da mesma no local. Todavia, o que muito se erra no planejamento urbano é a grande preocupação em conhecer sobre a espécie e a pequena preocupação em conhecer o ambiente em que se estará plantando ela.

A crescente substituição da flora nativa por plantas exóticas também pode ser considerada um problema, pois altera o ambiente natural que resta nos centros urbanos, uniformizando as paisagens de diferentes cidades e contribui para a redução da biodiversidade no meio urbano, dissociando-o do contexto ambiental onde se insere (MACHADO et al., 2006).

Os benefícios ambientais, sociais e econômicos da arborização urbana tornaram-se sem dúvida importantes temas de estudo. Diante dessa importância na qualidade de vida das pessoas e pela sua função na conservação da biodiversidade no meio urbano, esse trabalho teve como objetivo realizar um levantamento florístico, incluindo árvores, arvoretas e palmeiras presentes nas ruas e praças da cidade de Mata/RS, a fim de fornecer informações essenciais para ajudar os gestores em processos de decisão.

\section{MATERIAIS E MÉTODOS}

O estudo foi desenvolvido no município de Mata/RS que, geograficamente, localiza-se no centro do Estado do Rio Grande do Sul na zona da Depressão Central, integrada a Microrregião de Santa Maria, tendo como coordenadas geográficas: latitude - 29²7’29', Sul; longitude 54 27'29” Oeste; e altitude média de 127 metros, com aproximadamente 5,111 mil habitantes (IBGE, 2010). 
De acordo com a classificação climática de Köpen, o clima da região é caracterizado como Subtropical úmido do tipo Cfa, apresentando Temperatura média das máximas: $25^{\circ} \mathrm{C}$; Temperatura média: $20^{\circ} \mathrm{C}$; Temperatura média das mínimas: $13^{\circ} \mathrm{C}$. A precipitação média anual gira em torno de 1,4 mil milímetros. De acordo com a Classificação Cartográfica de Vegetação, a formação vegetal de Mata é caracterizada como Floresta Estacional Decidual.

O levantamento foi realizado nas vias públicas da cidade, dando importância para as praças e algumas ruas. As ruas estudadas foram aquelas que se apresentaram pavimentadas e com calçadas para pedestres, pois estas se apresentaram bem delineadas no que diz respeito às áreas privadas e vias públicas.

Cada árvore foi identificada individualmente e, com o auxílio de uma planilha, foram anotados dados como nome científico da espécie, nome vulgar e posição do espécime em um croqui área, método também utilizado por Souza et al. (2011). Os dados foram coletados no período de julho de 2007 a maio de 2008.

O levantamento de dados para a pesquisa foi realizado através de visitas in loco. A identificação das árvores foi realizada de maneira direta e imediata sempre que possível, utilizando para isso bibliografias específicas de apoio. Das espécies que não puderam ser identificadas no local foram retiradas amostras de ramos com folhas, flores e/ou frutos e/ou sementes disponíveis, para posterior e herborização. Foram fotografadas com máquina digital Fugi Finepix modelo S9100, levando-se em conta, ramo florido e planta inteira.

Após coletados, os dados, estes foram transcritos para uma planilha eletrônica utilizando o aplicativo Microsoft Excel, constando família, nome científico, categoria e quantidade. As espécies foram identificadas de acordo com o sistema de classificação APG III (2009).

A freqüência de cada espécie (\%) foi calculada através da razão entre o número de indivíduos da espécie e o número total de indivíduos do bairro. Os resultados obtidos foram plotados em gráficos e discutidos conforme bibliografia encontrada.

Para quantificar a diversidade de espécies optou-se por analisar dois componentes: riqueza, também chamada de densidade de espécies, baseada no número de espécies presentes; e uniformidade, baseada na abundância relativa de espécies e no grau de dominância ou falta desta, que segundo Odum (1988), são fundamentais no conceito de diversidade de espécies. Nesse sentido, realizou-se o índice de diversidade de Margalef (ROSSATO, TSUBOY e FREI, 2008) e índice de Uniformidade de Pielou para avaliar respectivamente a riqueza e uniformidade.

- Índice de Diversidade de Margalef, em que:

$$
\text { Alfa }=\frac{(S-1)}{\log N}
$$

Onde: Alfa = Índice de diversidade; $\mathrm{S}=$ número de espécies; $\mathrm{N}$ = número de indivíduos (COSTA, LINK e MEDINA, 2007).

Os valores inferiores a 2,0 são considerados como denotando áreas de baixa diversidade e valores superiores a 5,0 são considerados como indicador de grande biodiversidade.

- $\quad$ Índice de Uniformidade de Pielou (e) que é obtido através do índice de Diversidade de ShannonWeaver, onde:

$$
\theta=\frac{H^{t}}{\ln S^{t}}
$$

em que: $H^{\prime}=$ Índice de Shannon; $S^{\prime}=$ número de espécies.

- $\quad$ Índice de Diversidade de Shannon-Weaver (H’) é definido por:

$$
\begin{gathered}
H^{t}=-\sum_{t=1}^{s} p_{t} \ln p_{t} \\
\nu_{t}=\frac{n_{t}}{N}
\end{gathered}
$$

em que: pi= abundância relativa da i-ésima espécie; ni = número de indivíduos da i-ésima espécie; $\mathrm{N}=$ número total de indivíduos; $\mathrm{S}=$ número total de espécies. 


\section{RESULTADOS E DISCUSSÕES}

Neste trabalho foram identificadas 80 morfoespécies de plantas, entre cactos, árvores, arvoretas e palmeiras no ambiente urbano, distribuídas em 34 famílias num total de 1190 exemplares, conforme Tabela 1. Admite-se um mínimo de 7 a 10 espécies e recomenda-se um número de 10 a 20 espécies para compor a arborização de uma cidade (Milano e Dalcin, 2000).

Tabela 1 - Relação de espécies encontradas na arborização da cidade de Mata/RS, Brasil (2008) segundo a família, espécie, nome popular, o total de espécimes observados, origem e frequência relativa.

Table 1 - List of species present at urban forestry of Mata city / RS, Brazil (2008), according to family, species, popular name, overall number of species found, origin and relative frequency.

\begin{tabular}{|c|c|c|c|c|}
\hline Família & Nome Científico & Nome Popular & Ocorrência & $\begin{array}{l}\text { Freqüência } \\
\text { relativa (\%) }\end{array}$ \\
\hline \multirow[t]{3}{*}{ Anacardiaceae } & Mangifera indica $\mathrm{L}$. & mangueira & 1 & 0,08 \\
\hline & Schinus molle L. & aroeira-periquita & 14 & 1,18 \\
\hline & Schinus terenbinthifolia Raddi & aroeira-vermelha & 1 & 0,08 \\
\hline Annonaceae & Rollinia sylvatica (A.St. Hil.) Mart. & ariticum & 6 & 0,50 \\
\hline Apocynaceae & Nerium oleander L. & espirradeira & 1 & 0,08 \\
\hline Araliaceae & Schefflera arboricola Merr & schefflera & 5 & 0,42 \\
\hline \multirow[t]{2}{*}{ Araucariaceae } & Araucaria angustifolia (Bertol.) Kuntze & araucária & 2 & 0,17 \\
\hline & Araucaria columnaris (Forst.) Hooker & $\begin{array}{l}\text { pinheiro-de- } \\
\text { Natal }\end{array}$ & 1 & 0,08 \\
\hline \multirow[t]{6}{*}{ Bignoniaceae } & Jacaranda cuspidifolia Mart. & caroba-miúda & 5 & 0,42 \\
\hline & Jacaranda micrantha Cham. & caroba & 8 & 0,67 \\
\hline & Tabebuia alba (Cham.) Sandw. & $\begin{array}{l}\text { ipê-amarelo-da- } \\
\text { serra }\end{array}$ & 51 & 4,29 \\
\hline & Tabebuia avellanedae Lorentz ex Griseb. & ipê-roxo & 23 & 1,93 \\
\hline & Tabebuia chrysotricha (Mart. ex DC.) Standl. & ipê-amarelo & 18 & 1,51 \\
\hline & Tabebuia impetiginosa (Mart. ex DC.) Standl. & ipê-amarelo & 2 & 0,17 \\
\hline Bombacaceae & Chorisia speciosa A. St.-Hil. & paineira-rosa & 1 & 0,08 \\
\hline Boraginaceae & Patagonula americana $\mathrm{L}$. & guajuvira & 203 & 17,06 \\
\hline Cactaceae & Cereus peruvianus L. & cacto-do-peru & 1 & 0,08 \\
\hline \multirow[t]{3}{*}{ Cupressaceae } & Chamaecyparis obtusa (Siebold \& Zucc.) Endl. & cipreste-dourado & 1 & 0,08 \\
\hline & Thuja orientalis L. & cipreste & 3 & 0,25 \\
\hline & Thujopsis dolobrata (Thunb. ex L. f.) Siebold \& Zucc. & $\begin{array}{l}\text { falsa-árvore-da- } \\
\text { vida }\end{array}$ & 1 & 0,08 \\
\hline Juglandaceae & Carya illinoensis $\mathrm{K}$. & noz-pecã & 1 & 0,08 \\
\hline Lauraceae & Cinnamomum burmanni (Nees \& T.Nees) Blume. & falsa-canela & 4 & 0,34 \\
\hline \multirow[t]{7}{*}{$\begin{array}{l}\text { Leguminosae- } \\
\text { Caesalpinioideae }\end{array}$} & Caesalpinia peltophoroides Benth. & sibipiruna & 2 & 0,17 \\
\hline & Cassia ferruginea (Schrad) Schrad ex DC & chuva-de-ouro & 1 & 0,08 \\
\hline & Cassia leptophyllaVog. & falso-barbatimão & 1 & 0,08 \\
\hline & Hymenaeae courbaril L. & jatobá & 1 & 0,08 \\
\hline & Peltophorum dubium (Spreng.) Taub. & canafístula & 81 & 6,81 \\
\hline & Schizolobium parahyba (Vellozo) S. F. Blake. & guapuruvu & 1 & 0,08 \\
\hline & Senna macranthera H.S.Irwin \& Barneby & manduirana & 1 & 0,08 \\
\hline $\begin{array}{l}\text { Leguminosae- } \\
\text { Mimosoideae }\end{array}$ & Albizia polycephala (Benth.) Killip ex Record & angico-branco & 5 & 0,42 \\
\hline
\end{tabular}




\begin{tabular}{|c|c|c|c|c|}
\hline & Calliandra brevipes Benth. & esponjinha & 7 & 0,59 \\
\hline & Calliandra tweedii Benth. & ingá-do-brejo & 1 & 0,08 \\
\hline & Enterolobium contortisiliquum (Vell.)Morong. & timbaúva & 1 & 0,08 \\
\hline & Inga marginata Willd. & ingá-feijão & 35 & 2,94 \\
\hline \multirow[t]{2}{*}{$\begin{array}{l}\text { Leguminosae- } \\
\text { Papilionoideae }\end{array}$} & Erythrina crista-gali L. & $\begin{array}{l}\text { corticeira-do- } \\
\text { banhado }\end{array}$ & 1 & 0,08 \\
\hline & Tipuana tipu (Benth.) O.Kuntze. & tipuana & 4 & 0,34 \\
\hline Liliaceae & Yucca elephantipes Regel ex Trel. & Yuca & 2 & 0,17 \\
\hline Lytracea & Lagerstroemia indicaLam. & extremosa & 200 & 16,81 \\
\hline Magnoliaceae & Magnolia lilifloraDesr. & magnólia-roxa & 3 & 0,25 \\
\hline \multirow[t]{3}{*}{ Malvaceae } & Hibiscus mutabilis L. & flor-de-jericó & 1 & 0,08 \\
\hline & Hibiscus sinensisL. & $\begin{array}{l}\text { hibisco (rosa, } \\
\text { vermelho) }\end{array}$ & 3 & 0,25 \\
\hline & Hibiscus syriacus L. & hibiscus-da-síria & 2 & 0,17 \\
\hline \multirow[t]{2}{*}{ Meliaceae } & Cedrela fissilis Vell. & cedro & 35 & 2,94 \\
\hline & Melia azedarachL. & cinamomo & 105 & 8,82 \\
\hline \multirow[t]{2}{*}{ Moraceae } & Ficus enormis (Mart. ex Miq.) Miq. & $\begin{array}{l}\text { figueira-da- } \\
\text { pedra }\end{array}$ & 10 & 0,84 \\
\hline & Morus nigra L. & amoreira-preta & 17 & 1,43 \\
\hline \multirow[t]{9}{*}{ Myrtaceae } & Callistemon speciosus (Sims) Sweet. & $\begin{array}{l}\text { escova-de- } \\
\text { garrafa }\end{array}$ & 4 & 0,34 \\
\hline & Eucalyptus camaldulensis Dehn. & eucalipto & 2 & 0,17 \\
\hline & Eucalyptus urophila S.T. Blake. & melaleuca & 1 & 0,08 \\
\hline & Eugenia unifloraL. & pitangueira & 16 & 1,34 \\
\hline & Myrcianthes pungens (O.Berg) D. Legrand. & guabiju & 3 & 0,25 \\
\hline & Psidium cattleianum Sabine & araçá-vermelho & 6 & 0,50 \\
\hline & Psidium guajava pirifera $\mathrm{L}$. & goiaba-branca & 1 & 0,08 \\
\hline & Psidium guajava pomifera $\mathrm{L}$. & goiaba-vermelha & 4 & 0,34 \\
\hline & Syzygium cumini (L.) Skeels & jambolão & 3 & 0,25 \\
\hline Oleaceae & Ligustrum lucidum W.T. Aiton. & ligustro & 131 & 11,01 \\
\hline \multirow[t]{2}{*}{ Palmae (Arecacea) } & Butia paraguayensis (Barb. Rrodr.) L.H. Bailey & Butiá & 12 & 1,01 \\
\hline & Syagrus romanzoffiana (Cham.) Glassman & jerivá & 4 & 0,34 \\
\hline \multirow[t]{2}{*}{ Pinaceae } & Pinus echinata Mill. & pinus & 2 & 0,17 \\
\hline & Pinus elliotti Engel. & pinus & 3 & 0,25 \\
\hline Podocarpaceae & Podocarpus lambertii Klotzsch ex Endl. & pinheiro-bravo & 1 & 0,08 \\
\hline \multirow[t]{2}{*}{ Proteaceae } & Grevillea banksii R.Br. & grevilha-anã & 5 & 0,42 \\
\hline & Grevillea robusta A.Cunn. & grevilha & 1 & 0,08 \\
\hline Rhamnaceae & Hovenia dulcis Thunb. & uva-do-Japão & 12 & 1,01 \\
\hline \multirow[t]{2}{*}{ Rosaceae } & Eriobotrya japonica (Thunb.) Lindley & nespereira & 9 & 0,76 \\
\hline & Malus domestica Borkh. & macieira & 1 & 0,08 \\
\hline \multirow[t]{4}{*}{ Rutaceae } & Citrus aurantium L. & laranjeira-azeda & 9 & 0,76 \\
\hline & Citrus limonum Risso & limoeiro & 4 & 0,34 \\
\hline & Citrus sp & bergamoteira & 5 & 0,42 \\
\hline & Citrus $x$ sinensis (L.) Osbeck. & $\begin{array}{l}\text { laranjeira- } \\
\text { comum }\end{array}$ & 21 & 1,76 \\
\hline \multirow[t]{2}{*}{ Salicaceae } & Populus alba L. & álamo-prateado & 1 & 0,08 \\
\hline & Salix babylonica L. & chorão & 10 & 0,84 \\
\hline
\end{tabular}


Salix humboldtiana Willd.

Salix nigra Marsh.

Solanaceae

Sterculiaceae

Taxodiaceae

Tiliaceae

Verbenaceae salso

salso

romeu- e-julieta

perna-de-moça

pinheiro-alemão

açoita-cavalo-

miúdo

açoita-cavalo-

graúdo

pingo-d'ouro
0,84

0,08

0,08

0,50

0,08

2,02

0,08

0,17

Total $=20$

80

100

Legenda: * E - exótica, $\mathbf{N}$ - nativa

O índice de diversidade de Margalef representa 26,0. Este dado demonstra que o local inventariado possui uma elevada diversidade. Como não é recomendável a predominância de uma ou poucas espécies em arborização urbana, é conveniente manter-se uma boa variedade dos espécimes sempre seguindo a estética e observando-se o lado paisagístico (DANTAS e SOUZA, 2004). Contudo, nota-se que o número de espécies excedeu o recomendado.

Porém, para Cavalheiro (1995), a riqueza da vegetação pode se apresentar como indicador de qualidade ambiental em centros urbanos, representando inversamente o grau de alteração do ambiente. Acreditase, também, que uma variedade maior de espécies, em um ambiente urbano, promova melhor harmonia ecológica. Assim, novos habitats favorecem o surgimento de outras espécies, contribuindo para as interações ecológicas, entre elas, pode-se citar a competição, a predação e simbiose, dificultando o advento de pragas, as quais podem colocar em risco as populações.

Os valores no índice de uniformidade podem variar de $0 \mathrm{a}$ 1 , sendo que, quanto mais próximo de 1 , maior a uniformidade do local (KANIESKI, 2010), assim, para a área analisada o valor foi de 0,70 denotando boa uniformidade na composição da flora local.

Grey e Deneke (1978) recomendam, no planejamento da arborização urbana, frequências de 10 a 15\% do total da comunidade, para cada espécie utilizada. A justificativa seria a prevenção de riscos à longevidade, por meio de declínio e ataque de pragas e doenças. Conforme a recomendação observa-se que as freqüências de Patagonula americana L. (17,06\%) e Lagerstroemia indica Lam. (16,81\%) ultrapassaram o sugerido.

Ligustrum lucidum W.T. Aiton. foi a única espécie que apresentou a frequência recomendada $(11,01 \%)$, porém de acordo com Souza et.al. (2011), não é aconselhável a utilização dessa espécie como constituinte da flora urbana devido ao seu potencial tóxico.

Como pode ser visto na Figura 1, verificou-se que 57,5\% das espécies encontradas são classificadas como flora exótica e 42,5\% como flora nativa. Já quanto ao número individual de espécimes, há uma leve diferença, constando 49,2\% de indivíduos exóticos e 50,8\% de indivíduos nativos (índices visualizados na Figura 2).

A utilização de espécies exóticas na arborização urbana no Brasil é bastante comum (REZENDE e SANTOS, 2010). Como exemplo, cita-se a cidade de Maringá-PR, que está entre as mais arborizadas do país, onde 24,1\% das árvores são nativas (BLUM et al., 2008). É possível citar outros exemplos de frequência de espécies exóticas no ambiente urbano, como a cidade de Rio de Janeiro RJ (ZILLER, 2001), Pato Branco-PR (SILVA et al., 2007), Cachoeira do Sul-RS (LINDENMAIER e SANTOS, 2008), representando respectivamente 84,7\%; $60 \%$ e $58 \%$. 


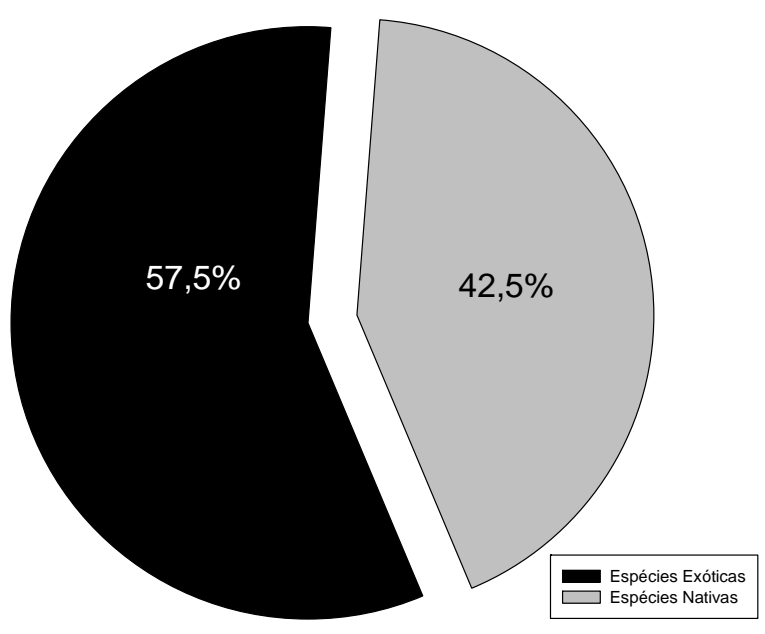

Figura 1 - Percentual de espécies exóticas e nativas na cidade de Mata/RS

Figure 1 - Percentage of exotic and native species at Mata's city/RS
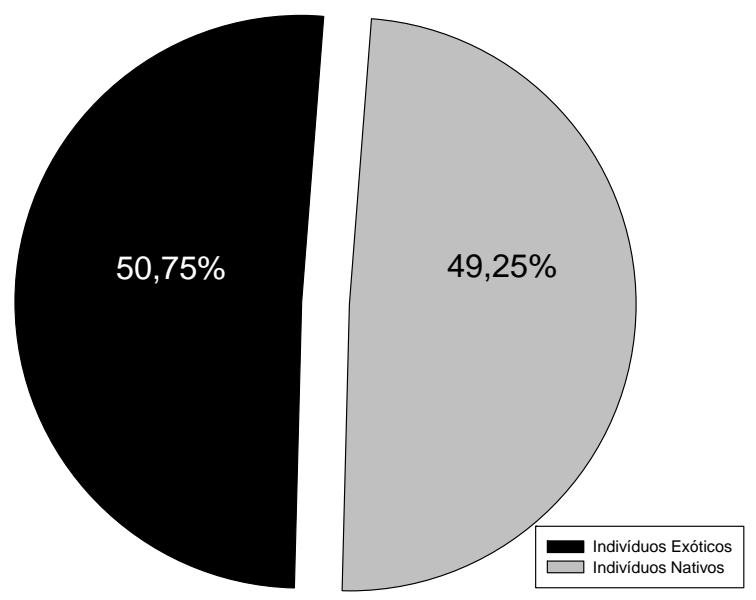

Figura 2 - Percentual de indivíduos exóticos e nativos na cidade de Mata/RS.

Figure 2 - Percentage of exotic and native individuals at Mata's city/RS.

\begin{abstract}
Matarazzo-Neuberger (1995) verificou que uma diversidade maior de espécies arbóreas consideradas nativas pode atrair para as áreas urbanizadas uma avifauna mais significativa. Cabe ainda lembrar que os pássaros não se alimentam somente de frutos, sendo necessário diversificar o quanto mais a vegetação, subsidiando a ornitofauna através das flores, seja pelo
\end{abstract}

néctar ou pelos insetos atraídos por ele. A qualidade, a quantidade e a distribuição das espécies arbóreas influenciam a fauna associada, contribuindo para a conservação da natureza (CAVALHEIRO, 1995).

As famílias botânicas que apresentaram destaque em número de espécies foram Myrtaceae, LeguminosaeCaesalpinioideae e Bignoniaceae, enquanto que as 
famílias com maior freqüência de indivíduos foram respectivamente Boraginaceae, Lytraceae e Meliaceae.

Fica visível que a cidade de Mata/RS é carente de cobertura vegetal, ao se tomar como referência a informação da United Nations Educational, Scientific and Cultural Organization (UNESCO), que recomenda a existência de pelo menos duas árvores ou cobertura de 12 $\mathrm{m}^{2}$ por habitante, para uma melhor qualidade de vida. Uma vez que a cidade possui um total de 5,111 mil habitantes, deduz-se que deveria abrigar 10,222 mil árvores. Os resultados observados anteriormente, porém, apontam para a existência de apenas 0,23 árvores para cada habitante. Naturalmente, esta taxa não corresponde à total realidade do município, visto que não foram contabilizadas as árvores presentes em jardins e quintais de residências. Contudo, mesmo que este número duplicasse, ainda assim estaria muito distante das exigências da UNESCO.

\section{CONSIDERAÇÕES FINAIS}

Observou-se uma grande riqueza de espécies e boa uniformidade na composição da flora local, porém, um baixo número de indivíduos na arborização da cidade em relação ao número de habitantes. Recomenda-se não dar continuidade ao plantio de Patagonula americana L. e, substituir ou evitar, o plantio de Lagerstroemia indica Lam., pois ambas ultrapassaram o limite sugerido pela bibliografia. Nesse sentido, nota-se a necessidade de um melhor planejamento da arborização e um remodelamento do aspecto paisagístico da cidade.

\section{REFERÊNCIAS BIBLIOGRÁFICAS}

AngiospermPhylogenyGroup (APG) III. An update of the Angiosperm Phylogeny Group classification for the orders and families of flowering plants: APG III Botanical. JournaloftheLinneanSociety, [s.n.], não paginado, 2009.

BLUM, C.T.; BORGO, M.; SAMPAIO, A.C.F. Espécies exóticas invasoras na arborização de vias públicas de Maringá-PR. Revista da Sociedade Brasileira de Arborização Urbana, Piracicaba - SP, v.3, n.2, p.78-97, 2008.

CAVALHEIRO, F. Urbanização e alterações ambientais. In: TAUK, S. M. Análise Ambiental: uma visão multidisciplinar. 2.ed. São Paulo: Editora da Universidade Estadual Paulista, 1995. p.114-124.

COSTA, E.C; LINK, D; MEDINA, L.D. de. Índice de Diversidade para entomofauna da Bragatinga (Mimosa scabrellaBenth.). Ciência Florestal, Santa Maria, v.3, n.1, p. 65-75, 1993.

DANTAS, I.C.; SOUZA, C.M.C. Arborização urbana na cidade de Campina Grande - PB: Inventário e suas espécies. Revista de biologia e ciências da terra, v. 4, n. 2, 2004.

GONÇALVES, E. O.; PAIVA, H.N.; GONÇALVES, W.; JACOVINE, L.A.G. Avaliação qualitativa de mudas destinadas à arborização urbana no Estado de Minas Gerais. Revista Árvore, v.28, n.4, p.479-486, 2004.

GONÇALVEZ, S.; ROCHA, F. T. Caracterização da arborização urbana do bairro de Vila Maria Baixa. Conscientia e Saúde, ano/vol2. Universidade Nove de Julho, São Paulo, Brasil, 2003, pp. 67-75.

GREY, G.W.; DENEKE, F.J. Urban forestry. New York, John Wiley, 1978.

INSTITUTO BRASILEIRO DE GEOGRAFIA E ESTATÍSTICA. 2010. Disponível em http://www.ibge.gov.br. Acesso em: 18 nov.2010.

KANIESKI, M.R. Caracterização florística, diversidade e correlação ambiental na Floresta Nacional de São Francisco de Paula, RS. Dissertação apresentada ao Curso de Mestrado em Engenharia Florestal, Universidade Federal de Santa Maria, Santa Maria, RS, Brasil, 2010.

LINDENMAIER, D.S. \& SANTOS, N.O. Arborização urbana das praças de Cachoeira do Sul - RS - Brasil: fitogeografia, diversidade e índices de áreas verdes. Pesquisas, Botânica, n. 59. p. 307-320. São Leopoldo. 2008. 
MACHADO, R. R. B., MEUNIER, I. M. J., SILVA, J. A. A., CASTRO, A. A. J. F. Árvores nativas para a arborização de Teresina, Piauí. Revista da Sociedade Brasileira de Arborização Urbana, Piracicaba - SP, v.1, n.1, p.10-18. 2006.

MAtARAZZO-NEUBERger, W. M. Comunidades de aves de cinco parques e praças da Grande São Paulo, Estado de São Paulo. Ararajuba, v.3, p13- 19, 1995.

MILANO, M. S.; DALCIN, E. C. Arborização de vias públicas. Rio de Janeiro: Light, 2000. 226p.

MOURA, T.A; SANTOS, V.L.L.V. Levantamento quali-quantitativo de espécies arbóreas e arbustivas na arborização viária urbana dos bairros centro e centro norte, Várzea Grande, Mato Grosso, Brasil. Revista da Sociedade Brasileira de Arborização Urbana, Piracicaba - SP, v.1, n.1, p.97-117, 2009.

ODUM, E. P. Ecologia. Rio de Janeiro: Guanabara Koogan, 1988. 434p.

REZENDE, T. M.; SANTOS, D.G. Avaliação quali-quantitativa da arborização das praças do bairro Jaraguá, Uberlândia MG. Revista da Sociedade Brasileira de Arborização Urbana, Piracicaba - SP, v.5, n.2, p.139-157, 2010.

RIBEIRO, F.A.B.S. Arborização urbana em Uberlândia: percepção da população. Revista da Católica, Uberlândia, v. 1, n. 1, p. 224-237, 2009

ROSSATO, D.R; TSUBOY, M.S.F; FREI, F. Arborização Urbana na cidade de Assis - SP: Uma abordagem quantitativa. Revista Sociedade Brasileira de Arborização Urbana, Piracicaba, v. 3, n.3, p. 1-16, dez. 2008.

SILVA, E.M.; SILVA, A.M.; MELO, P.H.; BORGES, S.S.; LIMA, S.C. Estudo da arborização urbana do Bairro Mansur na cidade de Uberlândia-MG. Caminhos de Geografia, v.3, n.5, p.73-83, 2002.

SILVA, L.M.; HASSE, I.; MOCCELIN, R.; ZBORALSKI, A.R. Arborização de vias públicas e a utilização de espécies exóticas: o caso do bairro centro de Pato Branco/PR. Scientia Agraria, vol. 8, núm. 1, 2007, pp. 47-53

SILVA, M.D.M.; SILVEIRA, R.R.; TEIXEIRA, M.I.J.G. Avaliação da arborização de vias públicas de uma área da região oeste da cidade de Franca/SP. Revista da Sociedade Brasileira de Arborização Urbana, Piracicaba, v.3, n.1, p. 19-35, mar. 2008.

SOUZA, A.R.C.; ROBAINA, A.D.R.; PEITER, M.X.; FERRAZ, R.C.; SCHWAB, N.T.; SOUZA, G.R.C.; PINTO, L.M. Identificação das espécies ornamentais nocivas na arborização urbana de Santiago/RS. Revista da Sociedade Brasileira de Arborização Urbana, Piracicaba - SP, v.6, n.2, p.44-56, 2011.

VOLPE-FILIK, A.; SILVA, L.F.; LIMA, A.M.P. Avaliação da arborização de ruas do bairro São Dimas na cidade de Piracicaba-SP através de parâmetros qualitativos. Revista da Sociedade Brasileira de Arborização urbana, v.2, n.1, p. 3443, 2007.

ZILLER, S. R. Plantas exóticas invasoras: a ameaça da contaminação biológica. Revista Ciência Hoje, Rio de Janeiro, n. 178, p. 77-79. dez. 2001. 